\title{
Germaine
}

\section{Avril Bradley}

In the greaseproof dish

of her manner she would

dry her words; a fat bag

of chipped phrases safeguard

against the oiliness of insincerity

straight from that arid zone

she would drive home

her hope for womanhood. 